\title{
Validez de contenido del modelo didáctico P-VIRC (preguntar- ver, interpretar, recorrer, contar) mediante el juicio de expertos
}

\author{
María S. Zamora-de-Ortiz¹, Francisca J. Serrano-Pastor² y María. J. Martínez-Segura² \\ (1) Universidad El Bosque, Facultad de Educación, Bogotá, Colombia (correo-e: zamoramaria@unbosque.edu.co) \\ (2) Universidad de Murcia, Facultad de Educación, Departamento de Métodos de Investigación y Diagnóstico en \\ Educación, Murcia, España (correo-e: fjserran@um.es, mjmarti@um.es)
}

Recibido Ago. 29, 2019; Aceptado Oct. 22, 2019; Versión final Dic. 17, 2019, Publicado Jun. 2020

\begin{abstract}
Resumen
El objetivo de esta investigación es analizar la validez de contenido del modelo didáctico P-VIRC (preguntarver, interpretar, recorrer, contar) por medio de la técnica estadística de juicio de expertos. Estos expertos evaluaron una escala diseñada ad hoc a fin de analizar cada una de las dimensiones y elementos del citado modelo, según los criterios de suficiencia, claridad, coherencia y relevancia. Este artículo evidencia los resultados de una investigación instrumental, con un diseño no experimental de carácter descriptivocomparativo. Los resultados evidencian que el modelo goza de validez de contenido y que existe concordancia entre los jueces en los criterios contrastados con la prueba W de Kendall. Se concluye que los componentes del modelo y sus indicadores de aprendizaje competencial instrumental, interpersonal y sistémico gozan de niveles de acuerdo satisfactorio entre los jueces, en los criterios de suficiencia, claridad, coherencia y relevancia.
\end{abstract}

Palabras clave: modelo educacional; competencias; educación superior; metodología estadística

\section{Content validity of the A-SIWT (ask, see, interpret, walk, tell) teaching model by expert judgment}

\begin{abstract}
The objective of this study is to analyze the content validity of the A-SIWT (ask, see, interpret, walk, tell) didactic model. This was performed using the statistical technique known as judgment of experts. Expert judges evaluated an ad hoc scale designed to analyze each of the dimensions and elements of A-SIWT by using the following criteria: sufficiency, clarity, coherence, and relevance. This article presents the results of an instrumental investigation that has a descriptive-comparative non-experimental design. The results show that the model has content validity and that there is agreement between the judges on the criteria contrasted with the Kendall W test. It is concluded that the components of the model and its indicators of instrumental, interpersonal, and systemic competence learning have satisfactory levels of agreement among judges in the criteria of sufficiency, clarity, coherence, and relevance.
\end{abstract}

Keywords: educational model; competence; higher education; statistic methodology 


\section{INTRODUCCIÓN}

El Modelo Preguntar-Ver, Interpretar, Recorrer, Contar (P-VIRC) es un modelo didáctico que pretende propiciar prácticas de aprendizaje significativo en el aula, a fin de que los estudiantes universitarios se comprometan con los problemas del momento histórico que están viviendo, a través de la generación de proyectos innovadores de aula, contemplados para desarrollarse en el semestre académico. En esa medida, este modelo permite modificar los procesos didácticos de las prácticas universitarias, desarrollando habilidades investigativas y comunicativas, así como la creatividad y la comprensión de la realidad. Para comprender el Modelo P-VIRC, se necesita conocer sus diferentes componentes: Preguntar, Ver, Interpretar, Recorrer y Contar. En primer lugar, a través de la "pregunta", se relaciona la teoría con la práctica y se transmite la percepción de la realidad. Este se considera el primero de los componentes del modelo, en el que comienza la tarea de investigación y se diseña una estrategia de búsqueda de la información. Al hablar de la relación entre teoría y práctica, nos referimos a la conjugación de aquellos saberes que se ponen en juego en el desarrollo de las competencias de los estudiantes, sintetizadas en los ejes: saber, hacer y ser. Este componente transversaliza las cinco dimensiones del modelo didáctico propuesto.

En cuanto al segundo componente "Ver", se comprende desde la perspectiva fisiológica hasta la percepción más compleja de la realidad, el modo de interpretarla y su expresión. En este orden de ideas, cuando se percibe una realidad o una fracción del mundo, "ver" se entiende como la forma de comprender la realidad. Por otra parte, "Interpretar" se refiere al papel que tiene el lenguaje en cuanto a la manera de construir la realidad. En seguida, el componente "recorrer" se relaciona con la habilidad del estudiante para comparar diferentes situaciones históricas, políticas, sociales y el recorrido por las mismas, que le lleva a una nueva reflexión propia sobre estas realidades. Finalmente, el componente "Contar" se establece como la posibilidad de transferir nuevos sentidos a los fenómenos al culminar el proceso. Así, el estudiante se enfrenta a un proceso creativo en el que imagina y en su interacción con los otros co-construye nuevos elementos para manifestar los conocimientos adquiridos en las etapas anteriores. La relación entre los 5 componentes del modelo P-VIRC se presenta en la figura 1.

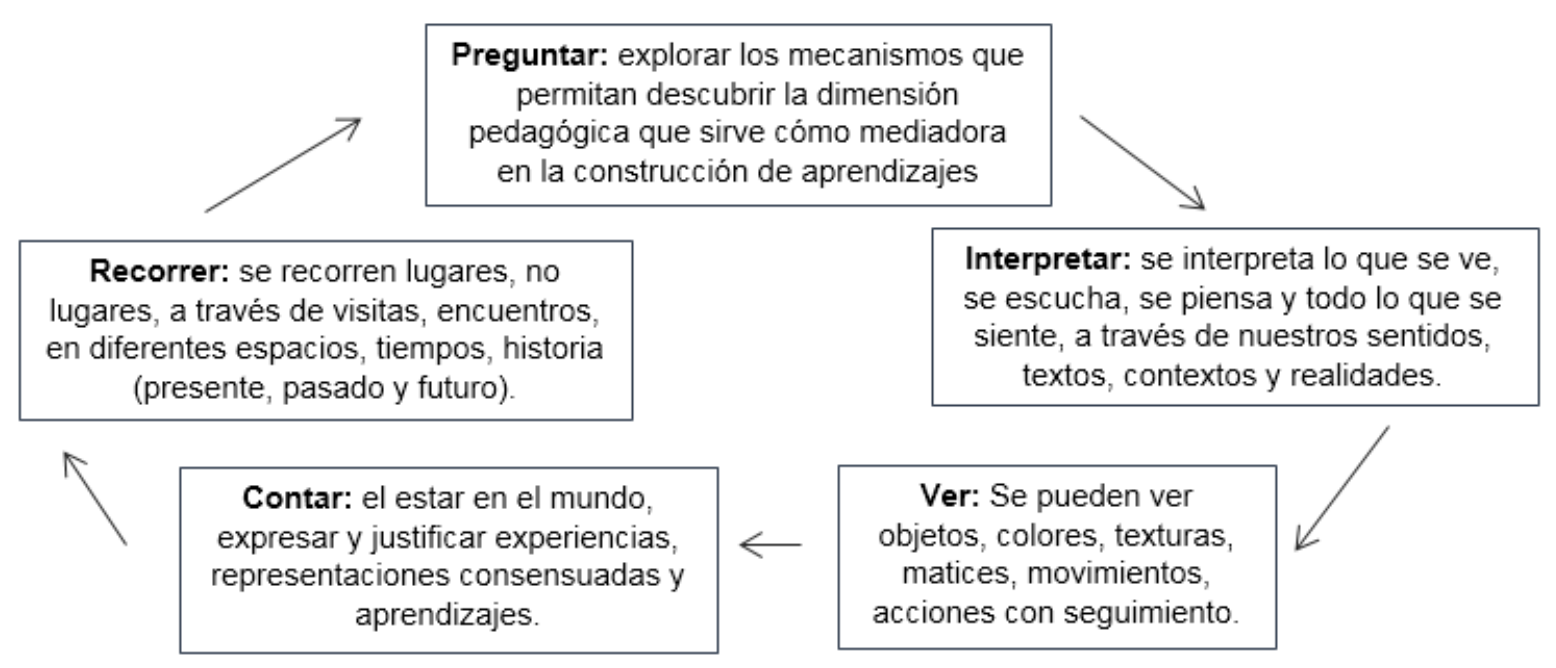

Fig. 1: Organización de componentes en el Modelo P-VIRC

Como se observa, la combinación de los componentes del Modelo P-VIRC conlleva a una articulación estrecha entre teoría y práctica, lo que favorece la mejora del aprendizaje. Lo anterior, relacionado con lo expuesto por Gordon (2009) desde la perspectiva del constructivismo pragmático, en la cual se señala que debe existir una interacción mutua entre teoría educativa y la práctica que desarrollan los profesores. El autor destaca que la actuación, la experiencia y el modelo didáctico que lleve el docente van a ser determinantes, en gran medida, de la calidad de los aprendizajes. Siempre que la teoría mantiene estrecha interacción con la práctica se puede apreciar una mejora en los aprendizajes (Raravi y Madhusudan, 2017; Wrenn y Wrenn, 2009).

Este modelo didáctico surge como una respuesta a la necesidad de propiciar un diálogo real entre la teoría y la práctica en las aulas universitarias, con el fin de promover una intervención activa de los estudiantes en su proceso de aprendizaje, a través de la puesta en práctica de su autonomía y de la capacidad de articular los saberes de la academia con los de su entorno social, laboral y personal. En esa medida, esta propuesta se alinea con otros ejercicios de investigación que plantean modelos didácticos adecuados para los contextos educativos actuales, como los realizados por Álvarez, Rodríguez-Pérez, Sanz-Ablanedo, y Fernández- 
Martínez (2008) en el que se propone un estudio basado en el aprendizaje autónomo y significativo de los educandos en un entorno de enseñanza aprendizaje activo. Y Tena (2010) en el que se aborda un estudio que destaca la importancia de la adquisición de competencias y la necesidad de evaluar el progreso de los estudiantes en dichas competencias utilizando instrumentos que permitan la mejora continua del aprendizaje.

Desde el 2010, el Modelo se implementó en la asignatura Práctica Comunitaria en el programa de "Licenciatura en Educación Infantil" de la Universidad El Bosque. El espacio de esta asignatura se presentó como el escenario ideal para desarrollar el modelo didáctico, ya que exige que los estudiantes se enfrenten como profesionales en formación a un ambiente real en el que deben poner en acción los conocimientos y saberes adquiridos hasta el momento. Desde entonces se ha trabajado en la consolidación conceptual y metodológica de dicho modelo, con el fin de mejorar los niveles de logros competenciales del alumnado. En los últimos años, el interés ha estado centrado en comprobar la validez de contenido del modelo didáctico para analizar si este refleja adecuadamente los constructos que está valorando. Acudimos a la validez de contenido, ya que este es el primer tipo de validez que debe garantizarse durante el diseño de una herramienta, considerándose un prerrequisito para el análisis de otros tipos de características métricas (Abdollahpour et al., 2011). Además, su estrecha relación con la confiabilidad del instrumento la colocan en una posición de vital relevancia en el proceso de construcción y validación de instrumentos. En ese orden de ideas, el presente artículo expone los resultados de la validez de contenido del modelo citado. Dicha investigación instrumental se acoge a un objetivo de una investigación más amplia que pretende el perfeccionamiento, la validación y la evaluación del modelo.

\section{Aspectos conceptuales}

El desarrollo del modelo P-VIRC se ha dado de manera paulatina. Inició como un esquema de clase en el 2010, que gradualmente se ha ido transformando de acuerdo con las necesidades que surgen de los procesos de enseñanza-aprendizaje y también con base en el resultado del quehacer docente y la experiencia en docencia universitaria. Se convierte en un proceso creativo que ha sido desarrollado a través de proyectos de aula y de las prácticas de los docentes en formación, en el que se brindan herramientas para utilizar aprendizajes concretos y para fortalecer el desarrollo de habilidades a nivel oral y de producción escrita de los estudiantes. Finalmente, los estudiantes alcanzan procesos conscientes de responsabilidad e identidad.

Para contextualizar el modelo, es necesario resaltar que para el caso colombiano el estudio de los Modelos Didácticos de Educación Superior es un hecho reciente y este interés se pone de manifiesto en numerosos trabajos (Gómez et al., 2016; Ortiz, A. et al., 2015). Hasta bien entrada la década de los ochenta, la formación universitaria colombiana estaba anclada en la práctica del modelo de enseñanza tradicional, que se limitaba a tres componentes básicos: el docente, el contenido y el estudiante. Con el paso del tiempo, hacia finales de la década de los noventa, las universidades comienzan a tener mayor autonomía, al tiempo se crean diferentes propuestas pedagógicas. Los supuestos teóricos basados en el conductismo, la enseñanza tradicional y el constructivismo proporcionaron los antecedentes que sirvieron para la transformación de la Educación Superior.

Pero ¿cuál es el concepto de modelo educativo en Educación Superior? En palabras de Tünnermann (2008) un modelo educativo sería "la concreción de los paradigmas educativos que una institución profesa y que sirve de referencia para todas las funciones que cumple (docencia, investigación, extensión, vinculación y servicios), a fin de hacer realidad su proyecto educativo". Además, debe sustentar la filosofía y finalidad de la institución. En ese orden de ideas, en este trabajo se tiene como principal referente el Modelo P-VIRC, que se sitúa en el punto de encuentro entre los modelos educativo, pedagógico y didáctico, como se observa en la Figura 2.

Como vemos, el modelo educativo se establece en la tensión entre el modelo pedagógico y el modelo didáctico; en este sentido, algunas posturas ubican a los modelos educativos en el contexto nacional, gestionados por los Ministerios de Educación y se basan en un paradigma aceptado por la comunidad científica. Por otra parte, en el modelo pedagógico se articulan las nociones de "currículo, pedagogía, didáctica, formación, educación, enseñanza, aprendizaje y evaluación; pero además contribuye a la configuración de procesos como la práctica y la teoría" (Ortiz et al., 2015). En lo que respecta al modelo didáctico, este se comprende como las acciones y estrategias empleadas por los profesores en el ejercicio de su profesión. Así pues, dicho modelo presenta "esquemas de la diversidad de acciones, técnicas y medios utilizados por los educadores" (Mayorga y Madrid, 2010). En conclusión, dicha interacción en los modelos genera una articulación entre la ciencia básica

La interacción de dichos modelos se centra en el currículo y en el Proyecto Educativo de la Institución, haciendo referencia a los diferentes aspectos que orientan los procesos curriculares. De acuerdo con Ortiz et al. (2015), "El modelo pedagógico se caracteriza por la articulación de nociones como: currículo, pedagogía, 
didáctica, formación, educación, enseñanza, aprendizaje y evaluación; además, contribuye a la configuración de procesos como la práctica y la teoría". Por último, "el modelo didáctico o de enseñanza presentan esquemas de la diversidad de acciones, técnicas y medios utilizados por los educadores" (Mayorga y Madrid, 2010).

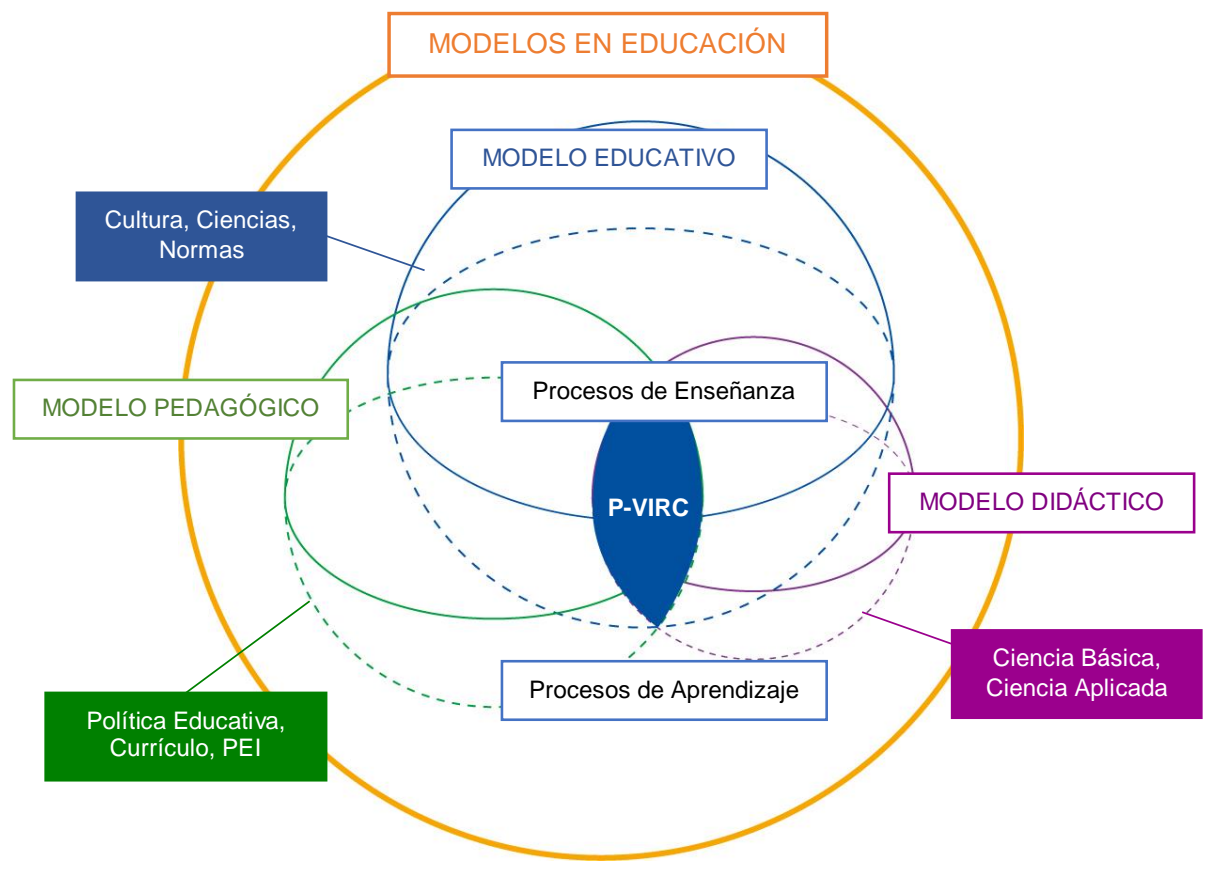

Fig. 2: Definición del Modelo didáctico P-VIRC como integración de modelos Pedagógico, Educativo y Didáctico

\section{Innovación y competencias}

Para fortalecer el desarrollo de la educación superior se ha propuesto el enfoque de formación por competencias, lo cual implica la interacción de diferentes saberes a fin de que sean aplicables y funcionales a la realidad personal y social que viven los educandos. De tal manera, las competencias refieren a procesos complejos de desempeño con idoneidad en determinados contextos, integrando diferentes saberes (saber ser, saber hacer, saber conocer y saber convivir), para realizar actividades y/o resolver problemas con sentido de reto, motivación, flexibilidad, creatividad, comprensión y emprendimiento (Tobón, 2008, p. 5).

Un importante reto en Educación Superior es proponer modelos que favorezcan situaciones de aprendizaje que lleven al estudiante a tener éxito académico, es decir, modelos que le permitan hacer del conocimiento una práctica real y significativa que consolide los tres saberes. Por ende, el trabajo por competencias es uno de los ejes principales que sustentan el modelo P-VIRC. Ya que al apoyar el proceso de enseñanzaaprendizaje en la adquisición de unas competencias, se contribuye a los estudiantes en su desarrollo como personas y como profesionales para un proyecto de formación que los va a acompañar a lo largo de toda su vida (Dresel, et al., 2015). De igual modo, las competencias integran de forma armónica, conocimientos, habilidades, actitudes y valores, ofreciendo garantías en la resolución de cualquier situación problemática en los diversos contextos en el que la persona se pueda encontrar (Tejada y Ruiz, 2016). En este perfil cobran especial relevancia las competencias instrumentales, interpersonales y sistémicas. Para Villa y Poblete (2008) las competencias instrumentales funcionan como un medio para conseguir un fin; son un conjunto de habilidades manuales y capacidades cognitivas que son necesarias para el desarrollo profesional. Por su parte, las competencias interpersonales demandan habilidades personales y relacionales que permiten objetivar, identificar y comunicar los sentimientos y emociones propias y los de los demás para fomentar la interacción social y la cooperación. Las competencias sistémicas son las destrezas y las habilidades que conciernen a los sistemas como totalidad; implican una combinación de imaginación, sensibilidad y habilidades que facilitan a la persona ver cómo las partes de un todo se unen y se relacionan, permitiéndole planificar cambios para la mejora y el diseño de nuevos sistemas. Estas competencias integradoras se desarrollan una vez que se ha adquirido las de carácter instrumental e interpersonal.

En el desarrollo de las competencias son primordiales las prácticas innovadoras, por ello la creación del Modelo Didáctico P-VIRC tiene como fin lograr diferentes caminos que den la posibilidad de proceder en términos pedagógicos, de manera nueva, para transformar los procesos de enseñanza desde la perspectiva del aprendizaje significativo. De tal forma, el modelo P-VIRC se establece con el objetivo de tener un nuevo recurso didáctico e innovador que permita ampliar las posibilidades de trabajo en el aula, es decir, que el 
modelo se convierta en una nueva herramienta para la transmisión del conocimiento. Sobre todo, en el contexto actual, en el que la tecnología ha influenciado todos los campos del saber del ser humano. Incluso la educación ha experimentado transformaciones importantes que han impactado esencialmente en las formas de desarrollar los procesos de enseñanza y aprendizaje, obligando a los actores a innovar para obtener mejores resultados y adaptarse a las necesidades de acuerdo con los cambios dados contextualmente.

Al respecto, Pérez-Gómez (2012) manifiesta que "La capacidad para usar las tecnologías de la información es cada día más determinante puesto que muchos de los servicios, trabajos e intercambios son y serán cada vez más accesibles solamente a través de la red. Por ello, aparece con mayor claridad y urgencia la necesidad de formación de los nuevos ciudadanos para vivir en un nuevo entorno digital de posibilidades y riesgos desconocidos" (p. 7). De esa manera, la innovación en el Modelo P-VIRC está mediada por el tratamiento de las herramientas ofrecidas por las tecnologías de la información en cada uno de sus niveles de desarrollo, es decir, observar, ver, interpretar, recorrer y contar. Finalmente, el modelo didáctico P-VIRC contribuye al desarrollo de unas competencias que satisfacen las necesidades y demandas del trabajo por competencias, potenciando las de tipo instrumental, interpersonal y sistémico. Es así como se garantiza una educación no solo significativa, sino que se alinea con el proyecto Tuning en Latinoamérica y Europa, el cual establece la necesidad de compatibilidad, comparabilidad y competitividad de la Educación Superior, pues actualmente la movilidad de los estudiantes es creciente y requiere de programas educativos fiables y objetivos (Villa y Poblete, 2008) que permitan la solidificación de unos saberes a través de la práctica de un modo consciente, ético y responsable.

\section{METODOLOGÍA}

El presente ejercicio investigativo es de orden instrumental (Ato et al., 2013), además, adopta un diseño no experimental de carácter descriptivo-comparativo, pues se propone el diseño y la validación de herramientas de medida. Particularmente, se propone analizar la validez de contenido de la adaptación del Modelo P-VIRC en la asignatura "Práctica Comunitaria" del programa de Licenciatura en Educación Infantil de la Universidad El Bosque de Bogotá, Colombia. En primer lugar, se realizó un proceso de adaptación de la asignatura mencionada al modelo P-VIRC, posteriormente se estableció una escala de valoración para llevar a cabo el juicio de expertos, lo cual arrojó unos resultados que se aplicarían en pro del mejoramiento del modelo. Como se observa en la figura 3.

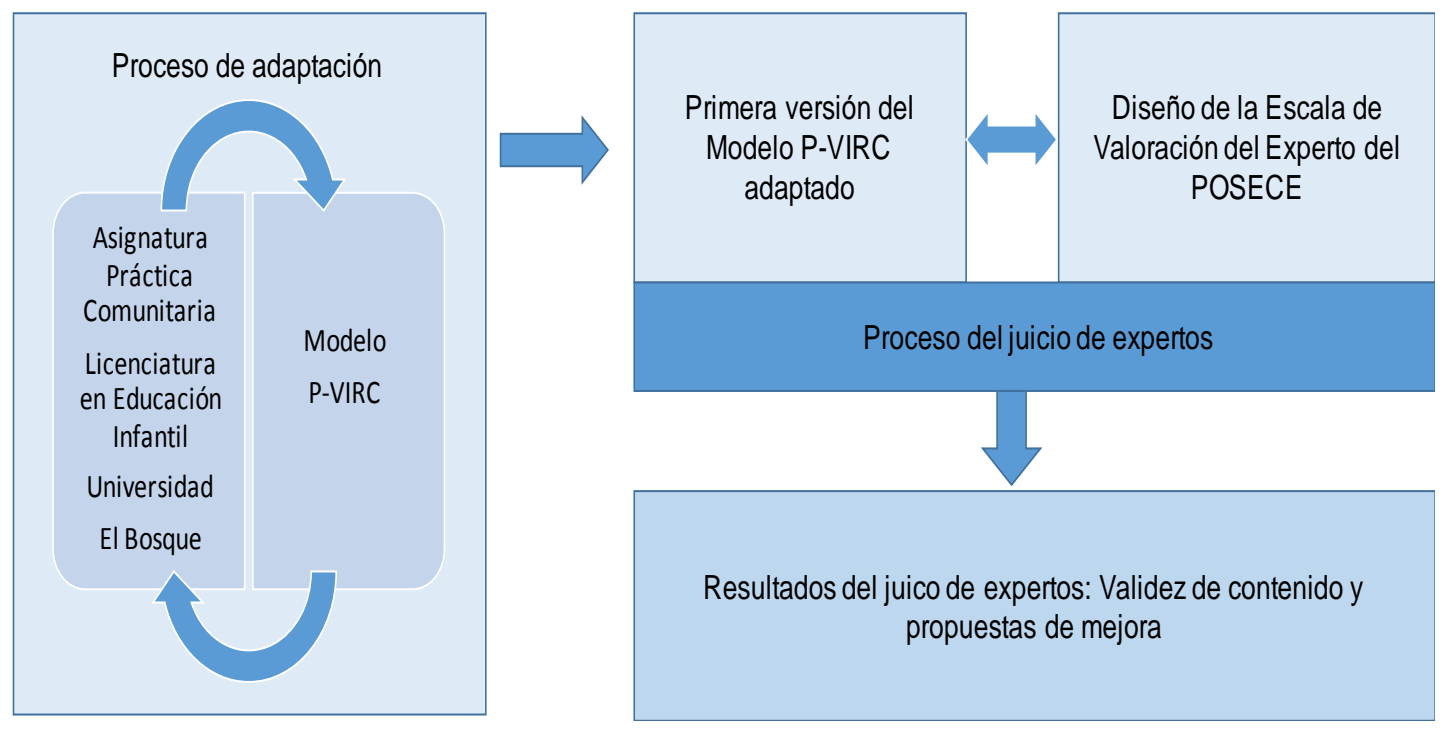

Fig. 3: Proceso de construcción y validación del Modelo P-VIRC

Para realizar el proceso de validación del modelo P-VIRC se incluyeron cada uno de sus componentes: Preguntar-Ver, Interpretar, Recorrer y Contar. El contenido de dicho modelo fue determinado a través de un Protocolo de Observación Sistemática para la evaluación de las Competencias del Estudiante (POSECE) que tuvo como propósito definir los criterios a evaluar y la escala de valoración. Los criterios seleccionados en el POSECE fueron suficiencia, claridad, coherencia y relevancia. El cruce entre estos criterios, las competencias del estudiante, dividas en tres grupos: instrumentales, interpersonales y sistémicas, y los componentes del modelo ya nombrados produjeron 18 ítems, sobre los cuales los jueces expertos debieron emitir sus juicios de valor u observaciones. La estructura de los criterios de valoración se describe en la Figura 4. 


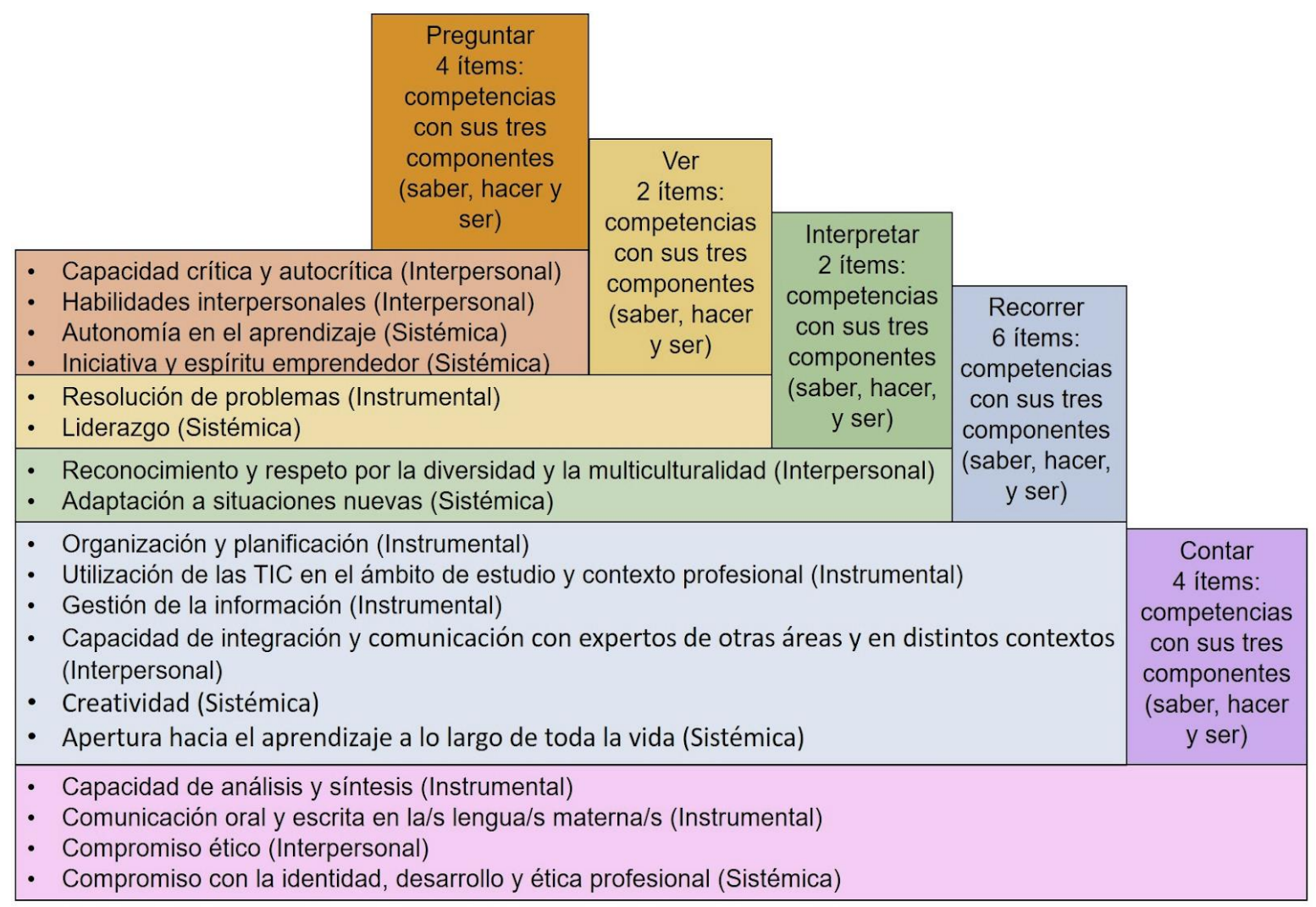

Fig. 4: Estructura del cuerpo de reactivos de la Escala de Valoración del Experto.

Ahora bien, el juicio de expertos es una estrategia frecuente para contrastar la validez de contenido donde se estima el grado de concordancia entre los expertos, en la que investigadores experimentados en el tema emiten sus valoraciones sobre los indicadores o ítems de la herramienta, según uno o más criterios (Bruna et al., 2019; Escobar y Cuervo, 2008; Souza et al., 2017; Wynd et al., 2003; Zamanzadeh, 2015). Así pues, este juicio se entiende como "una opinión informada de personas con trayectoria en el tema, que son reconocidas por otros como expertos cualificados en éste, y que pueden dar información, evidencia, juicios y valoraciones" (Escobar y Cuervo 2008, citado por Alarcón, Aidé, y Edel, 2017, p. 44).

La selección de expertos se realizó mediante un muestreo no probabilístico intencional o crítico (Taherdoost, 2016), así pues, hubo una selección acorde con la accesibilidad a la población, su disponibilidad e interés en ser parte de este ejercicio, su imparcialidad, independencia y experiencia docente e investigadora en el ámbito de la didáctica y la pedagogía en Educación Superior. De tal forma, se seleccionaron 18 especialistas, pero la muestra participante productora de información fue de 13, por lo que se produjo una mortandad experimental del $27.78 \%(n=5)$. Skjong y Wentworth (2001) señalan que la identificación de los especialistas es una parte muy relevante del proceso del juicio de expertos; de ahí que en la Tabla 1 se trace el perfil de los 13 jueces participantes, todos ellos con el grado de doctor, según sus características personales y profesionales.

Tabla 1: Perfil de los especialistas participantes en el proceso del juicio de expertos

\begin{tabular}{|l|l|r|r|}
\hline Característica & \multicolumn{1}{|c|}{ Frecuencia } & Porcentaje \\
\hline \multirow{3}{*}{ Género } & Hombre & 6 & 46.15 \\
\cline { 2 - 4 } & Mujer & 7 & 53.85 \\
\hline \multirow{3}{*}{ Universidad de procedencia } & Universidad de Murcia, España & 5 & 38.46 \\
\cline { 2 - 4 } & Universidad de El Bosque, Colombia & 7 & 53.85 \\
\cline { 2 - 4 } & Universidad La Salle, Colombia & 1 & 7.69 \\
\hline \multirow{2}{*}{ Categoría Profesional } & Catedrático de Universidad & 2 & 15.38 \\
\cline { 2 - 4 } & Profesor Titular de Universidad & 7 & 53.85 \\
\cline { 2 - 4 } & Profesor Asociado de Universidad & 4 & 30.77 \\
\hline Área de especialización & Gestión y organización educativa & 3 & 23.08 \\
\cline { 2 - 4 } & Métodos de investigación & 4 & 30.77 \\
\cline { 2 - 4 } & Diagnóstico en educación & 6 & 46.15 \\
\hline Experiencia docente e investigadora & De 6 a 17 años & 4 & 30.77 \\
\cline { 2 - 4 } & De 18 a 49 años & 9 & 69.23 \\
\hline
\end{tabular}


Antes de remitir la escala de valoración a los jueces, se les invitó a participar a través de un correo electrónico. Una vez que aceptaron, se les envió el instrumento indicándoles que disponían de 15 días para cumplimentarlo. Transcurrido este plazo, a aquellos investigadores docentes que no respondieron, se les recordó la tarea pendiente ampliando el plazo otra quincena. Cabe destacar que la información de los 13 expertos que colaboraron se obtuvo de manera individual sin la posibilidad de que pudieran interactuar entre ellos.

Como ya fue mencionado, y siguiendo las recomendaciones de Escobar y Cuervo (2008), los expertos debían emitir sus juicios en torno a los criterios de "suficiencia" (el ítem se corresponde con los resultados de aprendizaje que pretende evaluar o medir), "claridad" (el reactivo se comprende fácilmente, es decir, su sintáctica y semántica son adecuadas), "coherencia" (el elemento tiene relación lógica con el componente que está midiendo según el modelo P-VIRC), y "relevancia" (la competencia es esencial o importante, es decir, debe ser incluido en el modelo P-VIRC).

Para emitir sus juicios, los expertos utilizaron una escala ordinal con cuatro valores, en la que el 1 significaba "no cumple con el criterio" y el 4 "alto o total nivel de cumplimiento con el criterio". Cada uno de estos reactivos contó con un espacio abierto para que los jueces pudieran sugerir las modificaciones que considerasen pertinentes. Además, tuvieron la opción de proponer ítems adicionales o mejoras relevantes para el modelo. Este instrumento, de carácter digital, estuvo precedido de una "introducción o presentación" para dar a conocer a los jueces el propósito de la investigación en general y de la escala en particular, animarles a colaborar con su diligenciamiento, garantizarles el anonimato y la confidencialidad de la información aportada, informarles de la autoría de la investigación y de los datos de contacto de la misma y ofrecerles una explicación detallada del Modelo P-VIRC en el contexto didáctico en el que se debían realizar sus valoraciones. Para finalizar con el ejercicio, luego de los juicios generados por los jueces, se realizó una versión definitiva del modelo adaptado puntualmente a la asignatura de práctica comunitaria.

\section{RESULTADOS Y DISCUSÒN}

El análisis de los resultados ha supuesto, por un lado, el cálculo de los Índices de Validez de Contenido (CVI) a partir de la obtención de los coeficientes de Razón de Validez de Contenido (CVR') (Tristán, 2008, citado por Lawshe, 1975), de cada uno de los reactivos que conforman los cinco componentes del Modelo P-VIRC, según los cuatro criterios de evaluación valorados por los jueces (suficiencia, claridad, coherencia y relevancia). Por otra parte, se han calculado los coeficientes de concordancia entre jueces en los cuatro criterios y componentes citados mediante la prueba de rangos no paramétrica $W$ de Kendall para k muestras emparejadas (Siegel y Castellan, 1998), asumiendo el valor de significación $\alpha=0.05$ para contrastar las hipótesis de concordancia entre los expertos. Todas estas técnicas analíticas se han desarrollado con el programa estadístico IBM SPSS para Windows (versión 24).

EI CVI es una estrategia para estimar la proporción de acuerdo entre varios evaluadores que han juzgado el dominio del contenido representado en una herramienta (Lynn, 1986). Con el objetivo de poder calcular los CVR' de cada uno de los ítems -necesarios para estimar los CVI de los cinco componentes del modelo-, las puntuaciones asignadas por los panelistas, se recodificaron en dos categorías; de este modo, se consideró "contenido no válido" los valores 1 y 2 (incumplimiento o bajo nivel de cumplimento del criterio) y "contenido válido" los valores 3 y 4 de la misma (cumplimiento alto o total del criterio). Este procedimiento se ha utilizado en trabajos como los de Wynd et al (2003). La Tabla 2 muestra los CVR' de cada ítem en los criterios de suficiencia, claridad, coherencia y relevancia que han sido evaluados por los expertos, distribuidos en las cinco dimensiones del Modelo P-VIRC. Se observa que la totalidad de los reactivos son satisfactorios, de acuerdo con el criterio de Tristán (2008), para quien el valor de 0.5823 es el mínimo aceptable en un rango de 0 a 1 . Si se adopta el criterio de Abdollahpour et al. (2011), en las herramientas de nuevo diseño deben conservarse los reactivos con un valor igual o superior a 0.80 , siendo necesario mejorar aquellos ítems que están comprendidos entre 0.70 y 0.79 , y ser profundamente revisados o eliminados los menores de 0.70 .

En consecuencia, según este último criterio, todos los elementos de los cinco componentes del Modelo PVIRC deben conservarse si atendemos al criterio relevancia, ya que en los mismos existe al menos un $92 \%$ de acuerdo entre los jueces (CVR' iguales o superiores a 0.92) en que son esenciales o importantes debiendo ser incluidos según el modelo. En la dimensión Preguntar necesitan una mejora la claridad de los reactivos 1 y 2 (CVR'= 0.77), esto es, los indicadores de las competencias interpersonales "capacidad de crítica y de autocrítica" y "habilidades interpersonales". Del mismo modo, ha de revisarse la comprensión del elemento 7 del componente Interpretar que hace referencia a la competencia interpersonal "reconocimiento y respeto por la diversidad y multiculturalidad", así como las competencias 9, 10, 11 y 13 del componente Recorrer, especialmente la 10 y la 11 de carácter instrumental -"utilización de las TIC en el ámbito de estudio y contexto profesional" y "gestión de la información"-, ya que existe menos de un $70 \%$ de acuerdo entre los panelistas (CVR'= 0.69). 
Tabla 2: CVR' de los ítems del Modelo P-VIRC clasificados por componentes según los criterios valorados por los panelistas

\begin{tabular}{|l|c|c|c|c|c|}
\hline Componente del Modelo P-VIRC & Reactivo & $\begin{array}{c}\text { Suficiencia } \\
\text { CVR' }\end{array}$ & $\begin{array}{c}\text { Claridad } \\
\text { CVR' }\end{array}$ & $\begin{array}{c}\text { Coherencia } \\
\text { CVR' }\end{array}$ & $\begin{array}{c}\text { Relevancia } \\
\text { CVR' }^{\prime}\end{array}$ \\
\hline \multirow{4}{*}{ Preguntar } & 1 & 0.92 & 0.77 & 0.92 & 1.00 \\
\cline { 2 - 6 } & 2 & 0.84 & 0.77 & 0.84 & 0.92 \\
\cline { 2 - 6 } & 3 & 0.84 & 0.84 & 0.92 & 1.00 \\
\hline \multirow{4}{*}{ Ver } & 4 & 0.92 & 0.84 & 0.92 & 0.92 \\
\hline \multirow{5}{*}{ Interpretar } & 5 & 0.92 & 0.84 & 0.84 & 0.92 \\
\cline { 2 - 6 } & 6 & 0.84 & 0.84 & 0.92 & 0.92 \\
\hline \multirow{4}{*}{ Recorrer } & 7 & 0.84 & 0.77 & 0.84 & 1.00 \\
\cline { 2 - 6 } & 8 & 0.92 & 0.84 & 0.92 & 0.92 \\
\cline { 2 - 6 } & 10 & 0.84 & 0.77 & 0.92 & 1.00 \\
\cline { 2 - 6 } & 11 & 0.84 & 0.69 & 0.92 & 0.92 \\
\cline { 2 - 6 } & 12 & 0.84 & 0.84 & 0.92 & 1.00 \\
\cline { 2 - 6 } & 13 & 0.84 & 0.77 & 0.84 & 0.92 \\
\cline { 2 - 6 } & 14 & 0.84 & 0.84 & 0.92 & 0.92 \\
\hline Contar & 15 & 0.75 & 0.75 & 0.84 & 0.92 \\
\cline { 2 - 6 } & 16 & 0.92 & 0.83 & 0.92 & 1.00 \\
\cline { 2 - 6 } & 17 & 0.92 & 0.84 & 0.92 & 0.92 \\
\cline { 2 - 6 } & 18 & 0.92 & 0.84 & 0.92 & 0.92 \\
\hline
\end{tabular}

Los elementos 9 y 13 de este componente cuya redacción debe ser más adecuada (CVR'= 0.77) son: "organización y planificación" (instrumental) y "creatividad" (sistémica). También ha de mejorarse la coherencia de una de estas últimas competencias, "utilización de las TIC en el ámbito de estudio y contexto profesional" (ítem 10). El resto de los elementos se evidencian satisfactorios en cuanto que tienen relación lógica con el componente que está midiendo del modelo P-VIRC. Por último, se identificó un único elemento en el componente Contar que debe ser mejorado por no corresponderse completamente con los resultados de aprendizaje que pretende evaluar o medir (saber, ser/estar, hacer) al contar con un $75 \%$ de acuerdo entre los jueces (CVR'=0.75); se trata de la competencia instrumental "capacidad de análisis y síntesis" (reactivo 15).

Por tratarse el CVI del promedio de los ítems aceptables de acuerdo con el CVR', para Tristán (2008) se puede dictaminar la validez de contenido de un banco de reactivos tomando como valor mínimo 0.5823 que, como se ha comentado con anterioridad, es el límite inferior de los valores de CVR'. Pero, por la misma razón, la interpretación de estos índices puede hacerse según los criterios citados de Abdollahpour et al. (2011). Haciendo uso de ambos criterios, nos preguntamos ¿existe una proporción media de acuerdo entre los expertos en los bancos de elementos que conforman cada uno de los componentes del Modelo P-VIRC, que lleve a evidenciar que gozan de validez de contenido? La Figura 5 responde afirmativamente a esta cuestión, ya que en la misma se observa que para los cinco componentes y los cuatro criterios de evaluación, los CVI están comprendidos entre los valores 0.80 y 0.96 , lo que indica que ningún banco de reactivos debe eliminarse para aumentar su calidad. Únicamente el banco de ítems del componente Recorrer debería revisarse para que su sintaxis y su semántica fueran más adecuadas $(C V I=0.77)$. Estos resultados apoyan la lectura analítica individualizada que se ha realizado de los CVR' (Tabla 2) y nos llevan a concluir que se hace necesario revisar la redacción de los indicadores de las competencias del componente Recorrer del Modelo P-VIRC siguientes: "utilización de las TIC en el ámbito de estudio y contexto profesional", "gestión de la información", "organización y planificación" y "creatividad".

De forma complementaria al análisis cuantitativo de validez de contenido del Modelo P-VIRC, se han calculado los coeficientes $W$ de Kendall que indican el grado de asociación o nivel de concordancia entre los rangos promedios de las evaluaciones realizadas por los 13 expertos según una escala ordinal. Estas medidas se expresan en un rango de 0 a 1 , en el que el valor 0 representa una ausencia de concordancia y el 1 una total concordancia (Escobar y Cuervo, 2008; Siegel y Castellan, 1998). La prueba $W$ de Kendall además, ha permitido contrastar la hipótesis de la existencia de concordancia significativa entre los panelistas. 


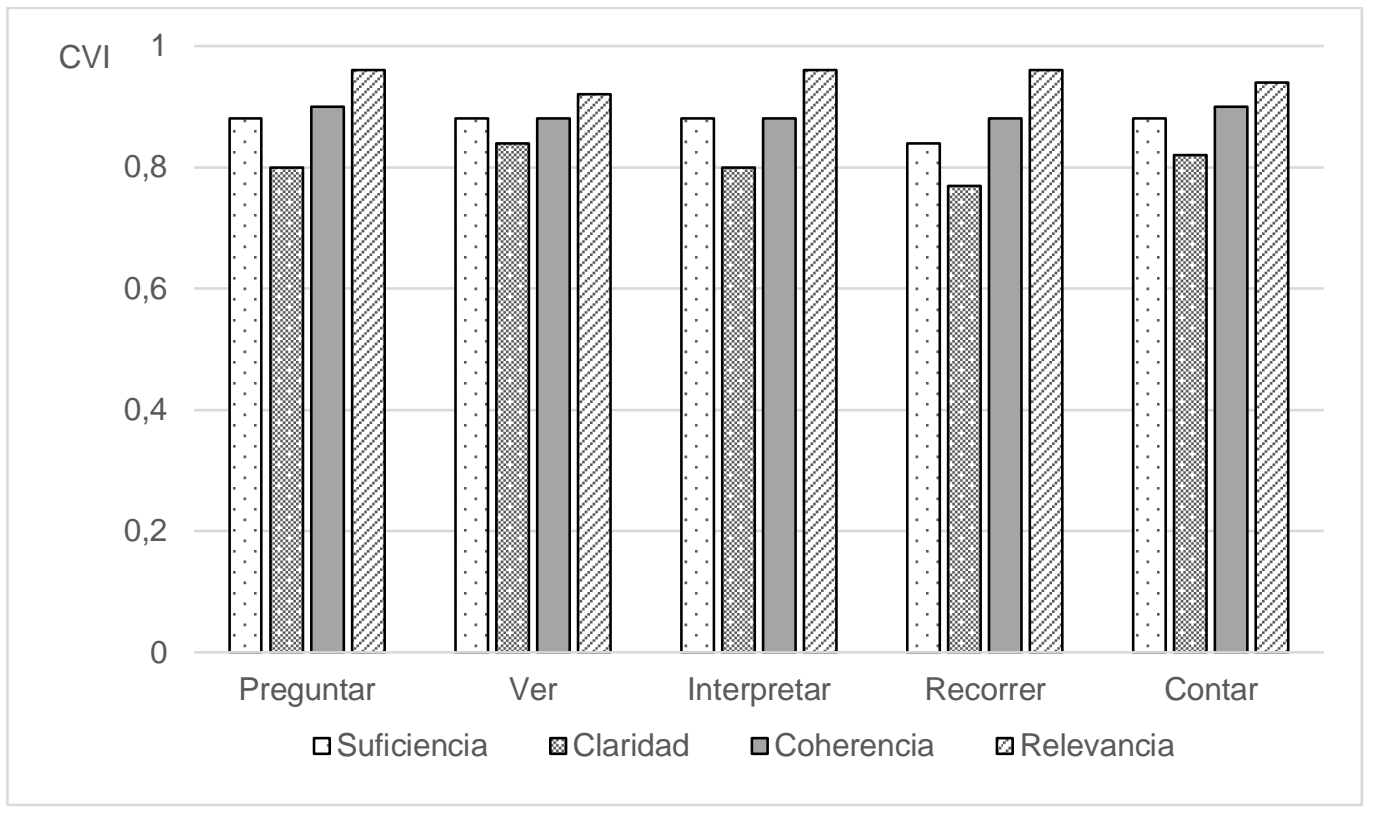

Fig. 5: CVI de los componentes del Modelo P-VIRC según los criterios valorados por los panelistas. Coeficientes de concordancia $W$ de Kendall

Los 20 contrastes realizados con la prueba $W$ de Kendall (Tabla 3 ), revelan que hay concordancia estadísticamente significativa entre los rangos promedios asignados por los jueces en relación con la suficiencia, claridad, coherencia y relevancia de las competencias del Modelo P-VIRC valorado ( $g l=12$, $p<.05)$. Esto es, los expertos concuerdan significativamente en sus juicios en que los indicadores de las competencias de cada componente del modelo: se corresponden con las tres dimensiones del resultado de aprendizaje que pretenden evaluar o medir (saber, saber ser y saber hacer); asimismo, se comprenden fácilmente, es decir, su sintaxis y semántica son adecuadas; tienen relación lógica con el componente del modelo que está midiendo y deben incluirse en la dimensión del modelo por ser esenciales.

Tabla 3: Estadísticos de la prueba W de Kendall de los componentes del Modelo P-VIRC según los criterios valorados por los panelistas

\begin{tabular}{|l|c|c|c|c|c|c|c|c|}
\hline \multirow{2}{*}{$\begin{array}{c}\text { Componente } \\
\text { Modelo P-VIRC }\end{array}$} & \multicolumn{2}{|c|}{ Suficiencia } & \multicolumn{2}{c|}{ Claridad } & \multicolumn{2}{c|}{ Coherencia } & \multicolumn{2}{c|}{ Relevancia } \\
\cline { 2 - 9 } & $W$ & $p$ & $W$ & $P$ & $W$ & $p$ & $W$ & $P$ \\
\hline Preguntar & 0.669 & 0.001 & 0.791 & 0.000 & 0.726 & 0.000 & 0.503 & 0.019 \\
\hline Ver & 0.994 & 0.021 & 0.938 & 0.037 & 0.687 & 0.027 & 0.895 & 0.044 \\
\hline Interpretar & 0.735 & 0.027 & 0.800 & 0.034 & 0.711 & 0.037 & 0.900 & 0.042 \\
\hline Recorrer & 0.732 & 0.000 & 0.739 & 0.000 & 0.601 & 0.000 & 0.596 & 0.000 \\
\hline Contar & 0.570 & 0.007 & 0.762 & 0.000 & 0.695 & 0.001 & 0.592 & 0.005 \\
\hline
\end{tabular}

Pero ¿cuál es la fuerza de la concordancia entre los expertos en cada uno de los componentes del Modelo P-VIRC según los criterios utilizados? El análisis individualizado de los coeficientes W de Kendall evidencia que están comprendidos entre los valores 0.503 y 0.994 (Figura 6), lo que 0020 indica que los grados de acuerdo oscilan entre moderados y muy altos. Los valores promedios de los coeficientes $\mathrm{W}$ de Kendall sitúan a los componentes Ver e Interpretar con los mayores niveles de acuerdo entre los evaluadores $\left(M W_{\text {ver }}=0.878\right.$, $M W_{\text {Interpretar }}=0.786$ ), frente a los promedios de los componentes Preguntar, Recorrer y Contar que se corresponden con las puntuaciones medias de $0.672,0.667$ y 0.638 , respectivamente.

Como vemos los resultados de las dos propuestas analíticas otorgan validez de contenido al Modelo P-VIRC según el juicio de los panelistas. Los indicadores de las competencias instrumentales, interpersonales y sistémicas de este modelo parecen tornarse en dominios de contenido esenciales, lógicos, fiables y objetivos, tal y como se demanda en el espacio de Educación Superior en Latinoamérica y Europa con la puesta en marcha del proyecto Tuning. Estas son características necesarias si queremos que los modelos de enseñanza-aprendizaje que se desarrollan en nuestros planes de estudio y asignaturas sean compatibles, comparables y competitivos (Villa y Poblete, 2008). 


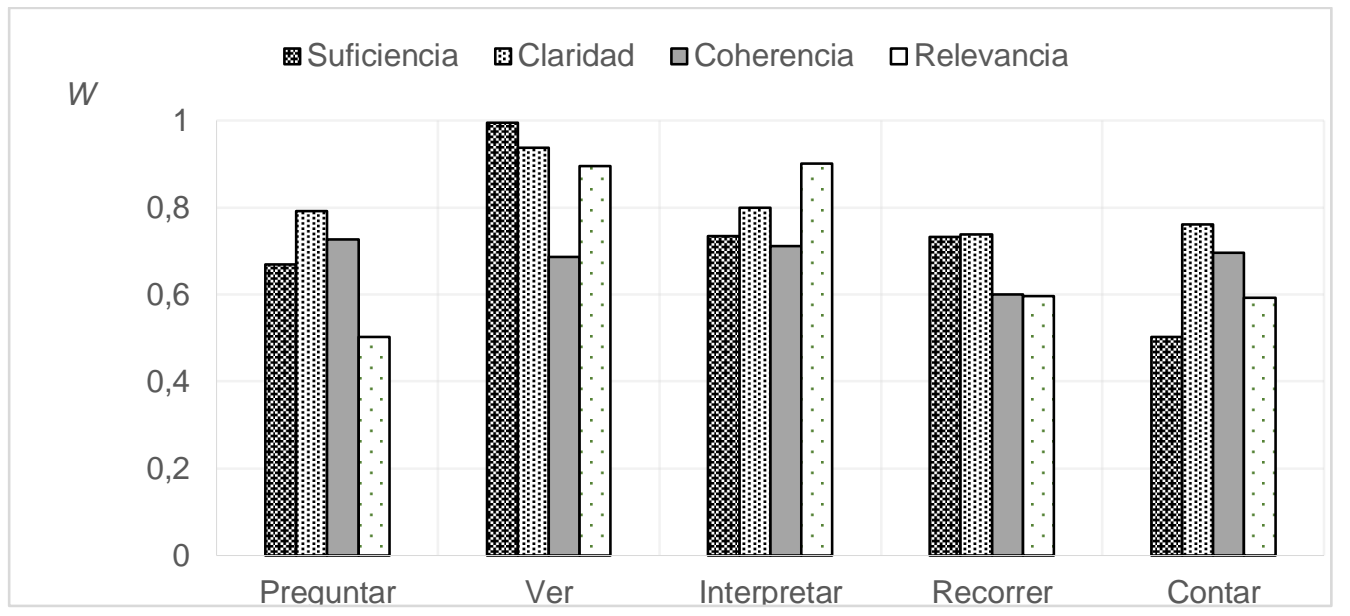

Fig. 6: Coeficientes de concordancia W de Kendall de los componentes del Modelo P-VIRC según los criterios valorados por los expertos

Se constata que pese a la revisión que se exige de algunos de sus reactivos, el Modelo P-VIRC se torna en una interesante y útil propuesta del aprendizaje basado en competencias (Biggs y Tang, 2011; Villa y Poblete, 2008), en el que se integran armónicamente habilidades, capacidades, conocimientos, actitudes y valores que permitan a los estudiantes prepararse para resolver las situaciones problemáticas que se le presenten en sus contextos académicos y profesionales a lo largo de sus vidas (Pallisera et al., 2010; Tejada y Ruiz, 2016). Igualmente, la articulación entre teoría y práctica que brindan los indicadores competenciales evaluados por los expertos del Modelo P-VIRC y que le dan su carácter holístico y sistémico a través de sus cinco componentes (Preguntar, Ver, Interpretar, Recorrer y Contar), refuerza el planteamiento constructivista pragmático en el que se basa (Gordon, 2009).

La validación de un modelo es un proceso largo y complejo. El estudio de su validez de contenido es el primer paso (Abdollahpour et al., 2011; Zamanzadeh et al., 2015). Garantizada la validez de contenido se deben analizar otras características como la confiabilidad, la validez del constructo y la criterial con datos recogidos en el desarrollo del modelo en el contexto curricular de la asignatura Práctica Comunitaria. La actuación y experiencia docente en interacción con las de los aprendices mediante el Modelo P-VIRC brindará la información necesaria que nos permita evaluar su rigor científico y, por tanto, determinar la mejora y calidad de los aprendizajes (Raravi y Madhusudan, 2017; Wrenn y Wrenn, 2009).

Cabe destacar algunas limitaciones de los estudios de validez de contenido mediante el juicio de expertos. Las valoraciones de los panelistas son subjetivas, por lo que los resultados de este tipo de estudios están sujetos a sesgos que pueden existir entre los mismos. En compensación a este posible sesgo, consideramos que los dominios de contenidos del modelo han estado bien identificados y operacionalizados a través de sus reactivos y que, ante la posibilidad de haber omitido alguno, se les pidió a los jueces que sugirieran otros elementos, lo cual no hicieron. Sus propuestas se han dirigido fundamentalmente a la mejora de la redacción de algunos de los resultados de aprendizaje vinculados con las competencias interpersonales del componente Recorrer del Modelo P-VIRC. Esto es, con aquellas que demandan al estudiante habilidades personales y relacionales que le permiten objetivar, identificar y comunicar sus propios sentimientos y emociones y los de los demás para fomentar la interacción social y la cooperación, y que son necesarias junto con las competencias instrumentales, para el desarrollo de las de carácter sistémico (Villa y Poblete, 2008) en el proceso de recorrer lugares, no lugares, a través de visitas, encuentros y experiencias de aprendizaje en diversos espacios, tiempo e historia.

Las limitaciones de sesgo de los panelistas también se han minimizado con su pericia en este tipo de tareas, con una trazabilidad homogénea de su perfil profesional y con el uso de la "Escala de Valoración del Experto del POSECE", en la que se han objetivado sus valoraciones mediante una medida ordinal con cuatro niveles bien delimitados, aspectos estos muy relevantes para autores como Bruna et al. (2019), Escobar y Cuervo, (2008), Skjong y Wentworth (2001), Souza et al. (2017), Wynd et al. (2003). Sin embargo, los jueces han hecho otra propuesta de mejora que se incorporará en la propia escala citada para su futura utilización en otros procesos de validación, como en el diseño de los instrumentos de evaluación de los niveles competenciales logrados por el alumnado en el proceso de enseñanza-aprendizaje con el Modelo P-VIRC en la asignatura Práctica Comunitaria. 


\section{DISCUSIÓN FINAL}

Como se explicó antes, el propósito de este trabajo ha sido analizar la validez de contenido, mediante el juicio de 13 expertos, del Modelo P-VIRC en el marco de la asignatura Práctica Comunitaria de la Licenciatura en Educación Infantil de la Universidad El Bosque de Bogotá, Colombia. En este contexto, los resultados muestran que los componentes del modelo y sus indicadores de aprendizaje competencial instrumental, interpersonal y sistémico gozan de unos niveles de acuerdo satisfactorios entre los jueces, en los criterios de suficiencia, claridad, coherencia y relevancia. Si bien, atendiendo a las orientaciones de Abdollahpour et al. (2011), debe revisarse la redacción de algunos reactivos, fundamentalmente los de las competencias interpersonales del componente Recorrer.

El desarrollo de dos procedimientos estadísticos, los CVI basados en el cálculo de los valores promedios de los CVR' y los coeficientes $W$ de Kendall con el consecuente contraste de las hipótesis de concordancia, se han complementado enriqueciendo el proceso para el estudio de la validez de contenido. Tras este análisis se evidencia que el modelo recoge los dominios de contenido representativos y esenciales, como primer paso necesario del largo y complejo proceso de validación que debe continuarse con la recogida de información durante su implantación en el ciclo de enseñanza y aprendizaje (confiabilidad, validez de constructo y validez criterial).

El sesgo de las valoraciones subjetivas de los panelistas se ha minimizado por la homogeneidad en sus perfiles profesionales y por la estructura y operacionalización de la escala de que han utilizado para emitir sus juicios. La validez de contenido del modelo es también la primera garantía de rigor científico para el diseño de instrumentos de evaluación de los aprendizajes de los estudiantes que se deriven del mismo, entre ellos el Protocolo de Observación Sistemática para la Evaluación de las Competencias del Estudiante (POSECE). El modelo se revela como una propuesta del aprendizaje basado en competencias, fiable y objetiva, como se demanda en el espacio de Educación Superior en Latinoamérica y Europa con la puesta en marcha del proyecto Tuning, y que la tornan en compatible, comparable y competitiva (Villa et al., 2008).

\section{CONCLUSIONES}

De acuerdo al trabajo presentado, al análisis desarrollado, y a los resultados obtenidos, se pueden plantear las siguientes conclusiones principales: 1) Los resultados muestran que los componentes del modelo y sus indicadores de aprendizaje competencial instrumental, interpersonal y sistémico gozan de unos niveles de acuerdo entre los jueces satisfactorios en los criterios suficiencia, claridad, coherencia y relevancia; 2) Atendiendo a las orientaciones de Abdollahpour et al. (2011), debe revisarse la redacción de algunos reactivos, fundamentalmente los de las competencias interpersonales del componente Recorrer; 3) El modelo recoge los dominios de contenido representativos y esenciales, como primer paso necesario del largo y complejo proceso de validación; 4) El sesgo de las valoraciones subjetivas de los panelistas se ha minimizado por la homogeneidad en sus perfiles profesionales y por la estructura y operacionalización de la escala de que han utilizado para emitir sus juicios; 5) El modelo se revela como una propuesta del aprendizaje basado en competencias, fiable y objetiva, como se demanda en el espacio de Educación Superior en Latinoamérica y Europa con la puesta en marcha del proyecto Tuning; 6) La aplicación del Modelo P-VIRC en la asignatura de Práctica Comunitaria, se podría considerar como un programa intracurricular (Sáez et al., 2018) que ayuda a promocionar unas competencias específicas de aprendizaje, en el contexto de una asignatura, dentro de un plan de estudios.

\section{REFERENCIAS}

Abdollahpour, I., Nedjat S., Noroozian M. y Majdzadeh R., Performing Content Validation Process in Development of Questionnaires, Iria. J. Epidemiol, 6(4), 66-74 (2011).

Álvarez F., Rodríguez-Pérez J.R., Sanz-Ablanedo E., Fernández-Martínez M., Aprender Enseñando: Elaboración de Materiales Didácticos que facilitan el Aprendizaje Autónomo. Formación Universitaria, 1(6), $19-28$ (2008).

Ato, M., López J.J. y Benavente A., A Classification System for Research Designs in Psychology, https://doi: 10.6018/analesps.29.3.178511, An Psicolo, 29(3), 1038-1059 (2013).

Biggs, J. y Tang C., Teaching for Quality Learning at University: What the Student Does, 4ª Ed., McGraw-Hill, Maidenhead, Berkshire (2011).

Bruna, C.E., Villarroel V.A., Bruna D.V. y Martínez J.A., Experiencia de Diseño y Uso de una Rúbrica para Evaluar Informes de Laboratorio en Formato Publicación Científica, https://doi: 10.4067/S0718-50062019000200017, Form. Univ, 12(2), 1728 (2019).

Galicia, L.A., Balderrama J.A. y Edel-Navarro R., Validez de Contenido por Juicio de Expertos: Propuesta de una Herramienta Virtual. Apertura (Guadalajara, Jal.), 9(2), 42-53. (2017). 
Gómez, J., Laverde H. y Díaz A., Tendencias Actuales de la Educación Superior en Colombia. https://doi: 10.15332/ s0124-3551.2016.0028.01, Revista CIFE, 18(28), 19-42 (2016).

Gordon, M., Toward A Pragmatic Discourse of Constructivism: Reflections on Lessons from Practice. https://doi: 10.1080/00131940802546894, Educational Studies, 45(1), 39-58 (2009).

Lawshe, C.H., A Quantitative Approach to Content Validity. https://doi: 10.1111/j.1744-6570.1975.tb01393.x, Personnel psychology., 28(4), 563-575 (1975).

Lynn, M.R., Determination and Quantification of Content Validity. https://doi: doi10.1097/00006199-198611000-00017, Nurs. Res., 35(6), 382-385 (1986).

Mayorga, M. y Madrid D., Modelos Didácticos y Estrategias de Enseñanza en el Espacio Europeo de Educación Superior, Tendencias Pedagógicas, 15(1), 91-111 (2010).

Newman, I., Lim J. y Pineda F., Content Validity Using a Mixed Methods Approach: Its Application and Development Through the Use of a Table of Specifications Methodology, https://doi: 10.1177/1558689813476922, J Mix Methods Res., 7(3), 243-260 (2013).

Ortiz, A., Sánchez J. y Sánchez I., Los Modelos Pedagógicos desde una Dimensión Psicológica-Espiritual, Revista Científica General José María Córdova, 13(15), 183-194 (2015).

Pallisera, M., Fullana J., Planas A. y Del-Valle A., La Adaptación al Espacio Europeo de Educación Superior en España, https://doi: 10.35362/rie5241784, Revista Iberoamericana de Educación Superior, 52(4), 1-13 (2010).

Pérez-Gómez, A. Educarse en la Era digital: Adelanto del Nuevo Libro de Ángel Pérez Gómez, Sinéctica [online], 40, 47$72(2013)$.

Raravi, P. y Madhusudan H.K., Enhancing Constructive Learning by Integrating Theory and Practice, https://doi: 10.16920/jeet/2017/v30i3/110611, JEET, 30(3), 340-345 (2017).

Sáez, F.M., Díaz A.E., Panadero E. y Bruna D.V., Revisión Sistemática sobre Competencias de Autorregulación del Aprendizaje en Estudiantes Universitarios y Programas Intracurriculares para su Promoción, https://doi: 10.4067/S071850062018000600083, Form. Univ., 11(6), 83-98 (2018).

Siegel, S. y Castellan N.J., Estadística no Paramétrica: Aplicada a las Ciencias de la Conducta, 4aㅡ Ed., Trillas, México (1995).

Skjong, R. y Wentworth B.H., Expert Judgment and Risk Perception, Eleventh International Offshore and Polar Engineering Conference, IV, 537-544 (2001).

Souza, A.C., Costa N.M. y Brito E., Psychometric Properties in Instruments Evaluation of Reliability and Validity, https://doi: 10.5123/S1679-49742017000300022, Epidemiol. Serv. Saude, 26(3), 649-659 (2017).

Taherdoost, H., Sampling Methods in Research Methodology; How to Choose a Sampling Technique for Research, https://doi: 10.2139/ssrn.3205035, Inter. J Academy. Resin. Manag., 5(2), 18-27 (2016).

Tejada, J. y Ruiz C., Evaluación de Competencias Profesionales en Educación Superior: Retos e Implicaciones, doi:10.5944/educXX1.12175, Revista UNED, 19(1), 17-38 (2016).

Tena, M. Aprendizaje de la Competencia Creatividad e Innovación en el Marco de una Titulación Adaptada al Espacio Europeo de Educación Superior. Form. Univ., 3(2), 11-20 (2010).

Tobón, S. La formación basada en competencias en la Educación Superior: el enfoque complejo, 2ª Ed, 1-28, CIFE, Guadalajara, México (2008).

Tristán-López, A., Modificación al Modelo de Lawshe para el Dictamen Cuantitativo de la Validez de Contenido de un Instrumento Objetivo, Avance en Mediación, 6(1), 37-48 (2008).

Tünnermann, C., Modelos Educativos y Académicos, Hispamer, Nicaragua (2008).

Villa, A. y Poblete M., Competence-Based Learning. A Proposal for the Assessment of Generic Competences. Universidad de Deusto, Bilbao, España (2008).

Wrenn, J. y Wrenn B., Enhacing Learning by Integrating Theory and Practice, IJTLHE, 21(2), 258-265 (2009).

Wynd, C.A., Schmidt B. y Schaefer M.A., Two Quantitative Approaches for Estimating Content Validity, https://doi: 10.1177/0193945903252998, Western J Nurs Res, 25(5), 508-518 (2003).

Zamanzadeh, V., Ghahramanian A. y otros cuatro autores. Design and Implementation Content Validity Study: Development of an Instrument for Measuring Patient-Centered Communication, https://doi: 10.15171/jcs.2015.017, J. Car. Sci., 4(2), 165-178 (2015). 